\title{
Le patrimoine documentaire comme soubassement d'un développement durable
} Nécessité de sa sauvegarde dans un établissement panafricain cinquantenaire

\section{Documentary Heritage as the Foundation for Sustainable Development}

\author{
The Need to Preserve it in Fifty-Year-Old Pan-African \\ Institution
}

\section{Antonin Benoît Diouf}

Volume 63, numéro 3, juillet-septembre 2017

Bibliothèques et développement durable : état des lieux

URI : https://id.erudit.org/iderudit/1041019ar

DOI : https://doi.org/10.7202/1041019ar

Aller au sommaire du numéro

\section{Éditeur(s)}

Association pour l'avancement des sciences et des techniques de la documentation (ASTED)

\section{ISSN}

0315-2340 (imprimé)

2291-8949 (numérique)

Découvrir la revue

\section{Citer cet article}

Diouf, A. B. (2017). Le patrimoine documentaire comme soubassement d'un développement durable : nécessité de sa sauvegarde dans un établissement panafricain cinquantenaire. Documentation et bibliothèques, 63(3), 18-23. https://doi.org/10.7202/1041019ar
Résumé de l'article

Le présent article vise à démontrer le lien qui existe entre les bibliothèques et le concept de développement durable. Il se concentre sur la nécessaire préservation du patrimoine documentaire pour favoriser un développement durable au bénéfice des générations futures. Le patrimoine documentaire a valeur de source d'inspiration pour toute action à venir et tout doit être mis en oeuvre pour que sa préservation soit durable pour une infinité temporelle. Cela est d'autant plus important dans un contexte africain où le développement représente le défi ultime de l'ensemble des pays du continent. L’argumentaire du présent article se base sur la réalité vécue dans une bibliothèque patrimoniale panafricaine confrontée à un sérieux problème de conservation de ses collections, une situation partagée avec bien d'autres structures documentaires du même type. Ses collections qui représentent une part de la mémoire gouvernementale africaine post-indépendante sont mises en péril par des agents de dégradation biologiques et physiques, hypothéquant ainsi leur accès par les futures ressources humaines d'Afrique.

Tout en montrant le lien évident entre accès à l'information et développement - comme l'a si bien compris l'IFLA, qui a imposé cet élément parmi les sous-objectifs des Objectifs du développement durable (ODD) -, l'exposé cherche aussi à montrer que les bibliothèques et les structures assimilées peuvent promouvoir le développement durable, non pas seulement en matière d'appropriation de techniques architecturales écologiquement rentables, mais aussi de comportements responsables dans la prise en compte des risques environnementaux. Pour ce faire, les bibliothèques doivent s'adapter aux législations nationales et transnationales en matière d'environnement pour conformer leurs services et modes de fonctionnement aux desiderata de ces dernières, ce qu'il est souvent difficile d'accomplir dans un contexte africain.
Tous droits réservés (c) Association pour l'avancement des sciences et des techniques de la documentation (ASTED), 2017
Ce document est protégé par la loi sur le droit d'auteur. L’utilisation des services d'Érudit (y compris la reproduction) est assujettie à sa politique d'utilisation que vous pouvez consulter en ligne.

https://apropos.erudit.org/fr/usagers/politique-dutilisation/ 


\title{
LE PATRIMOINE DOCUMENTAIRE COMME SOUBASSEMENT D'UN DÉVELOPPEMENT DURABLE:
}

\author{
nécessité de sa sauvegarde dans un établissement \\ panafricain cinquantenaire
}

\author{
Antonin Benoît DIOUF \\ Conservateur de bibliothèques \\ Chef des services de bibliothèque et de documentation \\ de l'Institut africain de développement économique \\ et de planification des Nations Unies (IDEP) de Dakar, Sénégal
}

antoninbenoit@gmail.com

Le présent article vise à démontrer le lien qui existe entre les bibliothèques et le concept de développement durable. II se concentre sur la nécessaire préservation du patrimoine documentaire pour favoriser un développement durable au bénéfice des générations futures. Le patrimoine documentaire a valeur de source d'inspiration pour toute action à venir et tout doit être mis en œuvre pour que sa préservation soit durable pour une infinité temporelle. Cela est d'autant plus important dans un contexte africain où le développement représente le défi ultime de l'ensemble des pays du continent.

L'argumentaire du présent article se base sur la réalité vécue dans une bibliothèque patrimoniale panafricaine confrontée à un sérieux problème de conservation de ses collections, une situation partagée avec bien d'autres structures documentaires du même type. Ses collections qui représentent une part de la mémoire gouvernementale africaine post-indépendante sont mises en péril par des agents de dégradation biologiques et physiques, hypothéquant ainsi leur accès par les futures ressources humaines d'Afrique.

Tout en montrant le lien évident entre accès à l'information et développement - comme l'a si bien compris I'IFLA, qui a imposé cet élément parmi les sous-objectifs des Objectifs du développement durable (ODD) -, l'exposé cherche aussi à montrer que les bibliothèques et les structures assimilées peuvent promouvoir le développement durable, non pas seulement en matière d'appropriation de techniques architecturales écologiquement rentables, mais aussi de comportements responsables dans la prise en compte des risques environnementaux. Pour ce faire, les bibliothèques doivent s'adapter aux législations nationales et transnationales en matière d'environnement pour conformer leurs services et modes de fonctionnement aux desiderata de ces dernières, ce qu'il est souvent difficile d'accomplir dans un contexte africain.

\section{Documentary Heritage as the Foundation for Sustainable Development: The Need to Preserve it in Fifty-Year-Old Pan-African Institution}

The following article aims to establish the relationship between libraries and the concept of sustainable development. It focuses on the necessary preservation of the documentary heritage in order to encourage sustainable development for future generations. Documentary heritage is a valuable source of inspiration for future actions and all efforts must be deployed to ensure that its preservation is durable for time to come. This is especially important in the African context where development represents the ultimate challenge for all the countries on the continent.

The argument put forward in this article is based on the experience of a pan-African heritage library faced with an important conservation challenge of its collections, a challenge shared with other libraries with similar collections. The collections in question represent a part of the government "memory" of postcolonial Africa and are deteriorating because of biological and physical agents, limiting their availability for future generations of Africans.

While the relationship between access to information and development is evident-a relationship understood by IFLA, that made this concern a part of the objectives of Sustainable Development Goals (SDG)-the article also illustrates that libraries and related organisations can promote sustainable development, not only with ecological architectural techniques, but also through responsible behaviour when assessing environmental risks. To achieve this, libraries must adapt their practices to the national and transnational environmental legislation and the desires or obligations of those legislations. This challenge is often difficult to achieve given the African context. 
D ès l'accession de la majorité des États africains à la souveraineté nationale, la problématique du développement s'est imposée à eux. L'Afrique héritière de siècles de domination matérialisée par la traite et la colonisation s'en trouvait bien mal partie, comme le soulignait d'ailleurs René Dumont dans son ouvrage éponyme (Dumont 1962). Il fallait dorénavant se prendre en charge, se substituer à la puissance coloniale qui conduisait les affaires des territoires anciennement annexés devenus subitement autonomes dans l'exercice d'une souveraineté pluridimensionnelle. Dès lors, une documentation politique, économique culturelle, sociale, etc. a commencé à être produite sous forme de documents de politique, notamment de plans de développement qui visaient à servir d'outils d'orientation pour gouverner ces «bateaux» ivres de leur jeunesse et de leur inexpérience dans la conduite d'entités territoriales généralement immenses avec des populations hétérogènes. Tant bien que mal, la plupart des États ont réussi le pari de la production intellectuelle pour soutenir leurs projets de gouvernements. Cette documentation constitue leur mémoire politique, un patrimoine qui doit être la source d'inspiration des projets actuels et futurs dont le seul but est d'accéder au développement comme aspiration légitime.

Cinquante ans ans après les fameuses indépendances, on note une situation catastrophique dans la conservation et la préservation de ce patrimoine, plus particulièrement dans les pays francophones anciennes colonies de la République française. Cela est lié à de nombreuses causes, dont l'absence de bibliothèques nationales ou de leur négligence de la part des décideurs politiques. Devant la carence de pareilles structures à l'échelle nationale, des initiatives à vocation transnationale se sont substituées tant bien que mal à elles et proposent aujourd'hui une option crédible dans l'exercice de conservation et de préservation normalement dévolue aux États souverains. Celle qui est l'objet de notre propos concerne l'Institut africain de développement économique et de planification des Nations Unies (IDEP), basé à Dakar avec une bibliothèque patrimoniale qui s'étend sur environ deux kilomètres de linéaires de documents et qui possède une collection qui a été rassemblée depuis 1963 et qui concerne au moins 49 pays africains soit environ 6000 titres de publications. Cette collection est constituée de plusieurs types de documents, tous relatifs aux politiques pensées ou mises en œuvre dans ces pays, qui constituent leur mémoire économique politique et culturelle, qui doit être la source d'inspiration de tout ce qui peut être pensé sur le plan du développement durable pour le présent et l'avenir. Cette collection est également dans un état de conservation très déplorable ce qui, à terme, l'expose à un risque de destruction certain. En effet, l'exposition à des facteurs de dégradation naturels est constante depuis l'aménagement du stockage au sous-sol et les conditions climatiques changeantes au cours des 50 dernières années sont venues s'ajouter aux causes de destruction. En outre, et pour peut-être prévenir la destruction des documents papier par des agents biologiques, les premiers administrateurs de la bibliothèque ont utilisé pendant longtemps des produits chimiques dont ils enduisaient les documents. Ces produits à base de mercure ont été depuis catalogués dangereux et nocifs pour l'homme et l'environnement. Le défi est donc double pour la bibliothèque pour ce qui est de sa relation avec le développement durable: d'une part, elle doit sauver un patrimoine de connaissances pour les générations futures et d'autre part elle doit corriger des erreurs humaines qui ont des effets directs sur la santé des personnes en contact avec ces documents traités avec des produits chimiques dont l'élimination pose un problème environnemental évident.

Le présent article fera état de tout cela et insistera plus particulièrement sur le diagnostic établi, l'identification des causes dégradantes et les solutions à court et long terme qui sont mis en œuvre ou qui sont planifiés pour préserver ce patrimoine en danger et l'environnement dans lequel il est conservé.

\section{La bibliothèque de l'IDEP}

Créé en 1962 par l'Assemblée générale des Nations Unies, l'IDEP est un organe de la Commission économique des Nations unies pour l'Afrique (CEA). Dès l'origine, son mandat a été d'accompagner et d'aider les pays africains nouvellement indépendants. Aujourd'hui, l'Institut propose aux 54 États membres divers services (formations, conférences, consultations, etc.) relatifs à tous les aspects multiformes du développement. 
La bibliothèque constitue un centre de ressources et de documentation important dans le domaine. Outre un fonds généraliste et classique en économie, les collections couvrent divers champs du développement économique, social et politique de l'Afrique postcoloniale sur des supports variés (monographies, brochures, périodiques, publications de grands organismes internationaux, ressources électroniques). Une récente opération d'identification des documents produits par les pays africains et conservés au sous-sol de la bibliothèque a permis de recenser environ 5800 titres de publications qui portent sur 49 pays. Cette collection n'est cependant qu'une infime partie de toutes celles stockées au sous-sol. Vu l'état de conservation, il est impératif de sauvegarder ces collections et de les protéger des nombreuses détériorations irréversibles qu'elles subissent actuellement.

\section{Diagnostic sur les causes de dégradation}

Cet exercice a pour objet de présenter l'état des collections en matière de conservation et de leur valorisation possible et de proposer une vue sur des solutions possibles au regard de ces deux éléments.

\section{Conservation}

Les conditions de conservation sont extrêmement mauvaises au sous-sol de la bibliothèque, ce qui nuit à la préservation des documents. La présence de nombreux agents destructeurs des documents a été observée: poussière, moisissures et insectes, inondations, produits toxiques, etc.

\section{Poussière}

La poussière est omniprésente sur l'ensemble des collections et le mobilier, et est favorisée par une situation géographique sahélienne, c'est-à-dire une zone de tampon entre le désert du Sahara et la zone équatoriale humide. La poussière s'avère être très nocive pour les documents, car elle favorise notamment le développement de moisissures. En l'absence de nettoyage régulier et en raison des ouvertures vers l'extérieur qui la laissent entrer, elle s'est agglutinée sur les documents jusqu'à constituer une couche compacte de quelques millimètres de hauteur certes, mais suffisants pour favoriser la détérioration. Le dépoussiérage pièce par pièce avec un aspirateur à filtre absolu est de rigueur pour nettoyer les documents ainsi que le mobilier et les magasins de conservation. Il faut également essayer de placer les documents à au moins deux ou trois centimètres à l'intérieur des rayonnages au moment du reclassement. En ce qui concerne le bâtiment lui-même, on envisage de calfeutrer les ouvertures vers l'extérieur et d'installer un système de ventilation qui filtre l'entrée de la poussière avec un système de nettoyage hebdomadaire des sols (balayage humide) et des rayonnages qui utilise des chiffons microfibres secs et propres, lavables à $90^{\circ}$ sans lessive ni adoucissant.

\section{Moisissures}

Divers types de moisissures ont été observés dans le soussol. Celles-ci sont favorisées par l'humidité importante, car «l'humidité est dangereuse par son action d'hydrolyse et favorisant en outre l'attaque biologique et la détérioration dues aux champignons, aux insectes, etc. (Pal Kathpalia 1973, 22). Les moisissures altèrent parfois de manière irréversible les documents. Elles sont causées sans doute par un système de climatisation défectueux et une absence de filtration de l'air, mais aussi par des infiltrations d'eau via les canalisations et la tuyauterie ainsi que les inondations épisodiques qui font suite aux fortes précipitations pluviométriques parfois notées dans la région dakaroise. Cela a d'ailleurs été le cas en 2011 lorsque le sous-sol de la bibliothèque a été inondé. Pour rattraper ces attaques dues aux moisissures, la procédure est de nettoyer les étagères des rayons, le sol et les entrées d'air avec de l'alcool à $70^{\circ}$. Ensuite, on doit procéder à une aspiration des documents par aspirateur à filtre absolu pour éviter la dispersion des spores, puis à une désinfection par prestataires extérieurs. Pour prévenir l'apparition des moisissures et la favorisation de leurs causes liées à l'humidité et la présence d'eau dans l'air, il est impératif d'exercer un contrôle climatique régulier en surveillant le taux d'humidité de l'air et la température à l'aide de procédés thermohygrométriques. La thermohygrométrie permet de mesurer le climat (température et taux d'humidité relative) et consiste en une évaluation des conditions climatiques d'une salle ou d'un bâtiment à l'aide d'instruments de mesure mécaniques ou électroniques disposés dans les salles et dont l'enregistrement peut se faire manuellement ou automatiquement, sur papier (graphique) ou sur ordinateur (valeurs numériques)ํ․

\section{Eau}

L'eau est un des facteurs de dégradation les plus présents dans le sous-sol de la bibliothèque en raison des inondations et infiltrations d'eau par le sol d'ordre naturel, mais aussi des fuites d'eau depuis les canalisations dues à l'ancienneté des conduits de canalisation. En ce qui concerne les inondations liées au climat - ce qui nous intéresse le plus ici -, elles sont causées par l'absence d'isolation dans le sol, dont la nappe phréatique est peu profonde, ce qui occasionne la submersion des étagères inférieures des rayonnages pendant la saison des pluies. En plus de favoriser l'apparition des moisissures décrites ci-dessus, cela a abîmé de nombreux rayonnages du sous-sol. Certains se sont même effondrés et ont provoqué la chute de documents et leur détérioration. Comme solution transitoire, on a procédé à l'installation d'une pompe pour évacuer et détourner l'eau vers des espaces plus appropriés (les jardins de

1. Guide interactif de conservation préventive: la thermohygrométrie. <www.conservationpreventive.be/site/la_thermohygrom_trie-301999-475.html> (consulté le 10/03/2017) 
l'Institut notamment) en attendant qu'un travail de surélévation du plancher du sous-sol soit réalisé.

\section{Toxicité}

Les documents de la bibliothèque ont longtemps été traités avec des produits toxiques. Cette approche se fonde sur des techniques de conservation anciennes préconisées avant les années 2000 qui favorisent l'utilisation de produits reconnus aujourd'hui très nocifs pour l'homme et pour l'environnement. C'est le cas par exemple de l'oxyde d'éthylène, qui agit comme fongicide, bactéricide et insecticide, et que l'on recommande pour désinfecter les documents d'archives (Fielder \& Duchein 1983, 70), mais qui s'avère potentiellement cancérigène pour l'homme (INRS 2016). La question délicate de l'élimination de ces produits toxiques hors de la bibliothèque se pose. Devant l'absence d'analyse de la teneur en mercure ${ }^{2}$ des documents et donc de l'absence d'éléments scientifiques de mesure de leur dangerosité, on doit instaurer la manipulation gantée des documents en guise de précaution. Il est impératif cependant de mettre à contribution des chimistes pour analyser le taux de toxicité des documents déjà traités au mercure et pour éliminer certains d'entre eux en fonction des résultats d'analyse. Nous devons également faire face au problème d'élimination desdits documents, mais aussi à celui du stock de produits restants conservé dans des bouteilles en verre qui risquent de se casser et de déverser les produits dans ces espaces de conservation. Il faut retenir les services de spécialistes pour procéder de manière adéquate à l'opération d'élimination en prenant en compte le facteur environnemental et en évitant que les produits ne polluent les espaces biologiques et ne contaminent les sources d'alimentation des êtres vivants.

\section{Préserver pour être le maillon fort dans la chaîne d'objectifs de développement durable}

Les Objectifs du développement durable (ODD) - ou l'Agenda 2030 - des Nations Unies ${ }^{3}$ en sont à leurs premiers pas depuis 2016 et les bibliothèques sont conscientes du rôle qu'elles doivent jouer dans ce mouvement mondial. L'IFLA (2002) l'affirme d'ailleurs en invitant les bibliothèques et les services d'information et leur personnel à soutenir et à promouvoir les principes du développement durable, et cela, depuis 2002. Cette prise de position précoce s'est réaffirmée

2. Une lecture du traité Convention de Minamata sur le mercure peut être source d'information et d'une meilleure compréhension de la nocivité de l'élément. La convention est disponible en ligne: <wedocs. unep.org/rest/bitstreams/14121/retrieve>

3. Nations Unies. 2015. Objectifs de développement durable: 17 objectifs pour transformer notre monde. <www.un.org/sustainabledevelopment/ fr/development-agenda/> (consulté le 11/03/2017) avec la participation des bibliothèques, en compagnie de plusieurs autres ONG, au Forum politique de haut niveau (High Level Political Forum ou HLPF), organisé en juillet 2015 à New York par les Nations Unies en préparation dudit Agenda 2030. À cet effet, l'IFLA a obtenu que l'on ajoute à l'objectif 16 la cible 16.10, qui consiste à: «Assurer l'accès public à l'information et protéger les libertés fondamentales, en accord avec la législation nationale et les accords internationaux.» (IFLA 2015). Dorénavant, le rôle des bibliothèques est bien défini et positionné dans l'architecture de la mise en œuvre et de la réussite des ODD. Toute bibliothèque doit dès lors s'inscrire dans cet impératif de garantir l'accès à l'information et, par extension, aux connaissances nécessaires au développement individuel et collectif.

\section{Patrimoine en danger: un frein certain à l'accès à l'information}

Dans le cas qui nous concerne, il est clair que la persistance de patrimoines en danger nuit à l'atteinte du sousobjectif 16.10 pour le développement durable des nations africaines. L'exemple de la bibliothèque de l'IDEP n'est malheureusement qu'une goutte d'eau dans un océan un peu navrant si on se réfère à des exemples plus célèbres comme celui des manuscrits de Tombouctou qui ont pu être «sauvés", ou encore aux collections inconnues ou méconnues de services d'archives publiques et privées qui ne sont pas dans les meilleurs états de conservation - par exemple les Archives Manyia du royaume Ashanti à Kumasi au Ghana ${ }^{4}$-, ou qui sont sujettes à des menaces de destruction par l'humain. En effet, outre les agents physiques et biologiques, dont les détériorations sont en quelques sorte «subies» et a priori impossibles à maîtriser, «l'homme peut également, d'une façon délibérée ou inconsciente, être à l'origine de très grands désastres: le vol, la guerre ou les émeutes, les manipulations trop brutales, l'application de techniques de conservation et de restauration inadaptées» (Flieder \& Duchein 1983, 25).

Ces fléaux d'ordre humain sont légion et ont accompagné l'histoire de l'Afrique postcoloniale, pour preuve l'incendie par des émeutiers des archives de l'Assemblée nationale au Burkina Faso en octobre 2014. Il faut mettre en œuvre des stratégies et d'autres plans d'action autour de la préservation du patrimoine documentaire africain. L'IFLA l'a aussi compris en encourageant et en parrainant la création de comités pour la préservation et l'accès (PAC) dans différentes régions du continent, dont la plus récente est celle consacrée à la sous-région francophone logée au Centre international de recherche et de documentation sur les traditions et les langues africaines (CERDOTOLA) ${ }^{5}$, basé à Yaoundé au Cameroun. Cette initiative est louable à tout

\footnotetext{
4. <www.manhyiaarchives.com/>

5. <www.pac-af.org/index.php/a-propos/missions/> (consulté le 11/03/2017)
} 
point de vue, car elle peut devenir un creuset d'échanges entre bibliothécaires et autres professionnels impliqués dans la préservation du patrimoine. Toutefois, son action ne pourra être plus efficace que si l'implication des décideurs politiques est de mise. Tant au plan national qu'international, des politiques et des actions qui visent à la préservation doivent être mieux institutionalisées et davantage entreprises sur le terrain en positionnant les bibliothèques et les structures assimilées au cœur de pareils dispositifs. Cela implique une plus grande allocation de ressources humaines, matérielles et financières. Pourquoi les bibliothécaires en seraient-ils les maillons forts? Parce que leur raison d'être est la médiation d'information et de savoirs et qu'avant de pouvoir jouer ce rôle de médiation, il faut avoir en sa possession le produit à diffuser et le formater de la manière la plus exploitable possible par l'usager (Courbières 2011).

Être au cœur du processus d'appropriation du patrimoine africain par l'usager africain, telle doit être la posture professionnelle de la bibliothèque africaine et de ses professionnels. Le développement durable passe en effet par le recours au passé utilisé comme référentiel politique, économique, social, culturel et scientifique pour éviter de «réinventer la roue» ou de tourner en rond quand il s'agit de penser et de conceptualiser le développement. Les leaders doivent s'imprégner des œuvres de leurs devanciers pour s'en inspirer dans un but d'améliorer les bonnes pratiques et d'éviter des erreurs constatées. Ils doivent aussi partager les plans de développement avec ceux pour qui ils sont élaborés et seront appliqués. Les chercheurs présents et ceux en devenir ont indéniablement besoin d'avoir accès à des documents primaires pour étayer des thèses et vérifier des hypothèses nécessaires à la production de savoirs «développementistes» et qui doivent être axés sur des contextes locaux. Les citoyens qui sont la majorité silencieuse des États, mais qui en constituent le socle par leurs activités multiformes et le lien social qu'ils cultivent, doivent être imprégnés de l'importance et de la valeur du patrimoine documentaire en tant que mémoire identitaire et bien commun à préserver.

Avec les ODD et leur dimension «accès à l'information", le professionnel de l'information documentaire voit ainsi ses missions se renforcer en tant que gardien de patrimoine, mais aussi en tant qu'éveilleur de conscience envers tous les acteurs sociaux, de manière à ce que l'on puisse éviter les manquements à la préservation d'ordre humain (volontaires ou involontaires) qui débouchent sur des pertes documentaires, et que tout un chacun se soucie de la sauvegarde du bien commun documentaire patrimonial.
Un des moyens d'y arriver est de s'inscrire dans une démarche de formation des utilisateurs active et proactive qui consiste à aller vers ces derniers, mais aussi à coupler celle-ci avec des actions de plaidoyer continu dirigées vers les décideurs politiques (État) à qui est déléguée l'autorité de faire appliquer des lois et des décisions. S'il réussit, ce plaidoyer permet d'avoir des cadres juridiques qui encadrent légalement la préservation du patrimoine, mais surtout qui exercent une coercition sur tous ceux qui enfreindraient ces cadres spécifiques.

\section{Préserver tout en participant à la culture d'un environnement sain}

En se basant sur l'exemple de la bibliothèque de l'IDEP exposé en première partie du présent texte, il est pertinent de discourir sur l'implication des structures documentaires patrimoniales dans la préservation d'un environnement vital sain et durable. Cette idée fait penser de prime à bord aux considérations écologiques à prendre en compte dans le choix des matériaux de construction des bibliothèques, par exemple (Henning s.d.). Cela est fort louable et de tendance depuis plus d'une dizaine d'années dans les cabinets d'architecture qui conçoivent des bibliothèques, mais le propos ici est d'une autre nature et lié à un vécu sans doute partagé par tous ceux qui gèrent des documents patrimoniaux. En effet, il s'agit davantage ici des risques liés à la pollution qui provient de la bibliothèque elle-même, lorsqu'il s'agit pour elle de se débarrasser de produits chimiques jadis utilisés pour contrecarrer l'action détériorante de certains agents biologiques sur le papier (champignons, moisissures, insectes). Avant que la recherche scientifique en général et médicale en particulier ne découvre l'effet nocif de certains produits pour l'environnement biologique (végétal et animal), les documents étaient traités par certains de ces produits pour les préserver contre la détérioration, notamment celle due aux agents biologiques listés ci-dessus. L'un des défis est de se débarrasser de ces produits, dont certains sont stockés après avoir été mis au contact avec les documents destinés à la manipulation humaine par les gestionnaires de ces documents, comme de potentiels utilisateurs. En l'absence évidente d'une formation en chimie, il est dangereux pour la majorité des professionnels qui travaillent dans ce patrimoine documentaire d'essayer de procéder à l'élimination de ces produits. Danger pour eux-mêmes, mais aussi danger pour l'environnement qui doit recueillir ces déchets avec les risques de contamination des éléments naturels que sont la terre, l'air et l'eau. Il faut donc forcément avoir recours à des textes d'ordre juridique pour connaître la conduite à tenir en pareil cas. Toutefois, dans un contexte africain, il 
n'est pas toujours aisé de trouver pareilles ressources et il est donc plus pertinent de se référer à des conventions internationales relatives aux produits chimiques. Il faudra ensuite voir, à l'échelle nationale, s'il y a des textes pour prendre en compte ces conventions dans le droit positif du pays en question.

Quelques indications peuvent être données sur ce plan, notamment celles de la Convention de Rotterdam sur la procédure de consentement préalable en connaissance de cause applicable à certains produits chimiques et pesticides dangereux qui font l'objet d'un commerce international, la Convention de Stockholm sur les Polluants organiques persistants et le Code d'éthique sur le commerce international des produits chimiques, pour n'en nommer que quelquesunes. Pour ce qui est du Sénégal, le pays a ratifié les différentes conventions ci-dessus et s'est doté d'un code de l'environnement qui fait l'objet de la Loi $n^{\circ} 2001-01$ datée du 15 janvier 2001. Il est le principal instrument juridique en matière de gestion des produits chimiques. Ce code est complété par le décret d'application $\mathrm{n}^{\circ}$ 2001-282 daté du 12 avril 2001 qui réglemente les modalités de gestion des produits chimiques ${ }^{6}$. La participation d'une structure documentaire patrimoniale à l'atteinte des ODD passe par une connaissance de pareilles dispositions juridiques liées à l'environnement afin de mieux y adapter certaines de ses activités pouvant compromettre l'équilibre naturel. Ce faisant, elle se donne aussi les moyens de vulgariser cette documentation juridique pour les usagers et fait ainsi une pierre deux coups dans la promotion du développement durable.

6. Sénégal. 2001. Décret n²001-282 du 12 avril 2001 portant Code de l'Environnement (partie réglementaire). 15 p. <http://www.rag.sn/ sites/rds.refer.sn/IMG/pdf/01-04-12CODENVIRONDECRET.pdf (consulté le 11/03/2017)

\section{Conclusion}

Les bibliothèques et les autres services d'information documentaire ont un lien évident avec le développement durable. L'effort intellectuel qui a été proposé ici visait à rendre compte de la nécessaire préservation du patrimoine documentaire pour favoriser la survenue d'un développement durable. Il a eu pour fondement argumentaire la réalité vécue dans une bibliothèque patrimoniale panafricaine confrontée à un sérieux problème de conservation de ses collections, collections qui représentent une part de la mémoire gouvernementale africaine post-indépendante et que des agents de dégradation biologiques et physiques mettent en péril, hypothéquant ainsi leur accès par les futures générations. Le but de l'exercice était de montrer le lien évident entre accès à l'information et développement, comme l'a si bien compris l'IFLA qui a imposé cet élément parmi les sous-objectifs des ODD. Le patrimoine documentaire a valeur de source d'inspiration pour toute action future et tout doit être mis en œuvre pour que sa préservation soit durable à très long terme. À côté de cette idée initiale, l'exposé a aussi voulu montrer que les bibliothèques et structures assimilées pouvaient promouvoir le développement durable, non pas seulement sur le plan de l'appropriation de techniques architecturales écologiquement rentables, mais aussi en matière de comportements responsables dans la prise en compte des risques environnementaux. En effet, les bibliothèques doivent se tenir au courant des législations nationales et transnationales en matière d'environnement pour que leurs services et modes de fonctionnement se conforment aux desiderata de ces dernières, ce qu'il est souvent difficile d'accomplir dans un contexte africain.

\section{SOURCES CONSULTÉES}

Baryla, Christiane. 2007. Poussière, moisissures et infestations dans les collections patrimoniales. Bulletin des bibliothèques de France (6): 106-107. <bbf.enssib.fr/consulter/bbf-2007-06-0106-014>.

Courbières, Caroline. 2011. L'analyse documentaire. In Approche de l'information-documentation: concepts fondateurs, sous la direction de Cécile Gardiès. Toulouse : Éditions Cépadues.

Coron, Sabine \& Lefèvre, Martine. 1993. Lutte contre les moisissures. Bulletin des bibliothèques de France (4): 45-52. <bbf.enssib.fr/ consulter/bbf-1993-04-0045-006>.

Dumont, René. 1962. L'Afrique noire est mal partie. Paris: Éditions du Seuil.

Flieder, Françoise \& Duchein, Michel. 1983. Livres et documents d'archives: sauvegarde et conservation. Paris: UNESCO, 70. <unesdoc.unesco.org/images/0005/000562/056224fo.pdf> (consulté le 10/03/2017).

Henning, Wolfram. s.d. La bibliothèque verte: écologie et durabilité dans la construction des bibliothèques. <www.adbdp.asso.fr/index. php/la-doc/cr-journees-d-etude/44-ouverture/94-la-bibliothequeverte-ecologie-et-durabilite-dans-la-construction-des-bibliotheques> (consulté le 14/03/2014).
IFLA. 2002. Déclaration des bibliothèques et du développement durable. $<$ www.ifla.org/files/assets/faife/publications/ifla-statement-sustainable-development-fr.pdf> (consulté le 11/03/2017).

IFLA. 2015. Boîte à outils: les bibliothèques et la mise en ceuvre de l'Agenda 2030 de l'ONU, Programme Action pour le développement à travers les bibliothèques. La Haye: IFLA.

IFLA PAC Francophone. 2017. <www.pac-af.org/index.php/a-propos/ missions/> (consulté le 11/03/2017).

INRS (France). 2016. Oxyde d'éthylène: Fiche toxicologique synthétique $n^{\circ}$ 70. Édition: Septembre 2016. Paris: INRS. <www.inrs.fr/ publications/bdd/fichetox/fiche.html ?refINRS=FICHETOX_70> (consulté le 10/03/2017).

Pal Kathpalia, Yash. 1973. Conservation et restauration des documents d'archives. Paris: UNESCO, 22. < unesdoc.unesco.org/images/0013/ 001376/137650fo.pdf > (consulté le 10/03/2017).

Laffont, Caroline \& Mouren, Raphaële. 2005. Les ennemis du livre. Bulletin des bibliothèques de France (1): 54-63. <bbf.enssib.fr/ consulter/bbf-2005-01-0054-013> (consulté le 10/03/2017).

Nations Unies. 2015. Objectifs de développement durable: 17 objectifs pour transformer notre monde. <www.un.org/sustainable development/fr/development-agenda/> (consulté le 11/03/2017). 